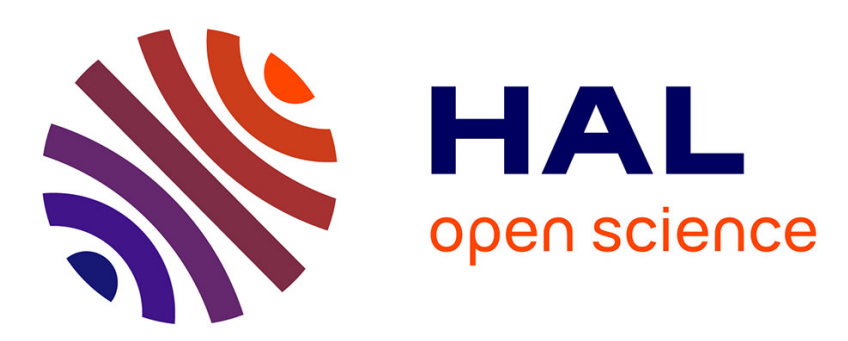

\title{
Bistability of a compliant cavity induced by acoustic radiation pressure
}

Bruno Issenmann, Régis Wunenburger, Sébastien Manneville, Jean-Pierre

Delville

\section{- To cite this version:}

Bruno Issenmann, Régis Wunenburger, Sébastien Manneville, Jean-Pierre Delville. Bistability of a compliant cavity induced by acoustic radiation pressure. Physical Review Letters, 2006, 97 (7), pp.074502. 10.1103/PhysRevLett.97.074502 . ensl-00527171

\section{HAL Id: ensl-00527171 \\ https://hal-ens-lyon.archives-ouvertes.fr/ensl-00527171}

Submitted on 18 Oct 2010

HAL is a multi-disciplinary open access archive for the deposit and dissemination of scientific research documents, whether they are published or not. The documents may come from teaching and research institutions in France or abroad, or from public or private research centers.
L'archive ouverte pluridisciplinaire HAL, est destinée au dépôt et à la diffusion de documents scientifiques de niveau recherche, publiés ou non, émanant des établissements d'enseignement et de recherche français ou étrangers, des laboratoires publics ou privés. 


\title{
Bistability of a Compliant Cavity Induced by Acoustic Radiation Pressure
}

\author{
Bruno Issenmann, ${ }^{1}$ Régis Wunenburger, ${ }^{1, *}$ Sébastien Manneville, ${ }^{2}$ and Jean-Pierre Delville ${ }^{1}$ \\ ${ }^{1}$ Centre de Physique Moléculaire Optique et Hertzienne, UMR CNRS 5798, \\ Université Bordeaux I, 351 cours de la Libération, 33405 Talence Cedex, France \\ ${ }^{2}$ Centre de Recherche Paul Pascal, UPR CNRS 8641, Avenue Schweitzer, 33600 Pessac, France
}

\begin{abstract}
We report on the first observation of multiple-order bistability due to acoustic radiation pressure in a compliant acoustic cavity formed between a spherical ultrasonic transducer immersed in water and the free liquid surface located at its focus. The hysteretic behavior of the cavity length, observed both with amplitude ramps and frequency sweeps, is accurately described using a one dimensional model of compliant Fabry-Pérot resonator assuming the acoustic radiation pressure to be the only coupling between the cavity and the acoustic field.
\end{abstract}

PACS numbers: 43.25.+y, 47.20.Ma, 47.20.Ky

The coupling between the path length of a Fabry-Pérot resonator and the intensity of the light travelling back and forth inside it leads to a rich variety of steady and dynamic nonlinear phenomena, e.g. bistability, regenerative oscillations, monostable pulse generation, and chaos [1]. The optical path may vary either from an intensitydependent refractive index of the material filling the resonator (bulk nonlinearities), or from the variation of the actual distance between the mirrors due to their interaction with light (boundary nonlinearities) through several mechanisms, among them radiation pressure (RP). Although RP based mechanism for inducing optical bistability of compliant Fabry-Pérot resonators have been evidenced in the 1980's [2, 3], RP has recently attracted a renewed attention for two main reasons: (i) the miniaturization down to the microscale of optical resonators used in nano or quantum detectors that increases their compliance and makes boundary nonlinearities dominant over bulk nonlinearities [4-6], and (ii) the high laser power required in the extremely compliant, vacuum filled interferometric gravitational waves detectors (GWD). Indeed, if on one hand, RP has been shown to allow for an efficient control of the position, stiffness and signal to noise ratio of microlevers [5], on the other hand, $\mathrm{RP}$ is likely to alter the response of the GWD interferometers $[7,8]$. Thus, the ambiguous role played by RP in these new applications fields motivates new research effort on its effects.

Although the above listed nonlinear phenomena have been mainly studied using electromagnetic waves, similar manifestations of the coupling between a wave and a resonator have also been observed using mechanical waves $[9,10]$. Bistability of high amplitude acoustic waves standing in rigid closed cavities has been reported [9], due to the amplitude dependence of the wave velocity arising from bulk nonlinearities [11]. Surprisingly, while acoustic RP has been evidenced for a long time $[12,13]$ and is the basis of several processes $[14,15]$ and measurement techniques $[16,17]$, to our knowledge, it has never been

\footnotetext{
*Corresponding author: r.wunenburger@cpmoh.u-bordeaux1.fr
}

used to trigger the bistability of compliant resonators. Still, the assets of using acoustics to study the physics of waves are mainly (i) the low frequencies and the large wavelengths involved, which allow for a direct measurement of the phase of the waves and of the path length, (ii) the strength of the acoustic RP, which makes its mechanical effects noticeable at the macroscopic scale, even at moderate acoustic intensity and focusing. Finally, such an investigation addresses the question of the universality of the mechanical effects of the RP (i.e. of their independence with respect to the nature of the exciting wave), which remains a matter of intense debate $[18,19]$.

In this Letter, we report on the first observation of multiple-order bistability in a compliant Fabry-Pérot resonator solely due to the acoustic RP. The cavity, sketched in Fig. 1a, is filled with water and is bounded on one side by an immersed spherical ultrasonic transducer emitting vertically and on the other side by the perfectly reflecting free surface of water (intensity transmission coefficient of $10^{-3}$ at normal incidence) located at the focus of the transducer. We measure the deformations of the free surface induced by RP as function of the input acoustic power and frequency, and using a simple one dimensional model of Fabry-Pérot cavity, we demonstrate that RP is actually responsible for the observed bistability.

A spherical Imasonic ultrasonic transducer (UT, focal length $F=38 \mathrm{~mm}$, aperture $N A=1, f=2.25 \mathrm{MHz}$ central frequency, $600 \mathrm{kHz}$ bandwidth, focal zone length $z=4 \mathrm{~mm}, 5 \mathrm{~s}$ maximal sonication time), bored with a cylindrical hole of diameter $2 \mathrm{~mm}$ along its axis and filled with a glass cylinder, is immersed vertically in pure, degased water contained in a transparent tank, see Fig. 1a. It is fed by an Amplifier Research $75 A 250$ power amplifier (PA) driven by a PC-controlled waveform generator (WG). The instantaneous amplitude of the WG output signal is extracted using an amplitude demodulator, and sent to a digital oscilloscope. Independent pressure measurements in a large water tank showed that in the focal plane the amplitude $P_{0}(r)$ of the pressure field $p_{0}(r, t)$ due to the beam emitted by the UT (hereafter called input beam) is well described by the usual expression for spherical, non bored transducers [20] $P_{0}(r)=P\left|J_{1}(\pi r / \lambda) /(\pi r / 2 \lambda)\right|$, as shown in Fig. 1c $\left(J_{i}\right.$ is 
(a)

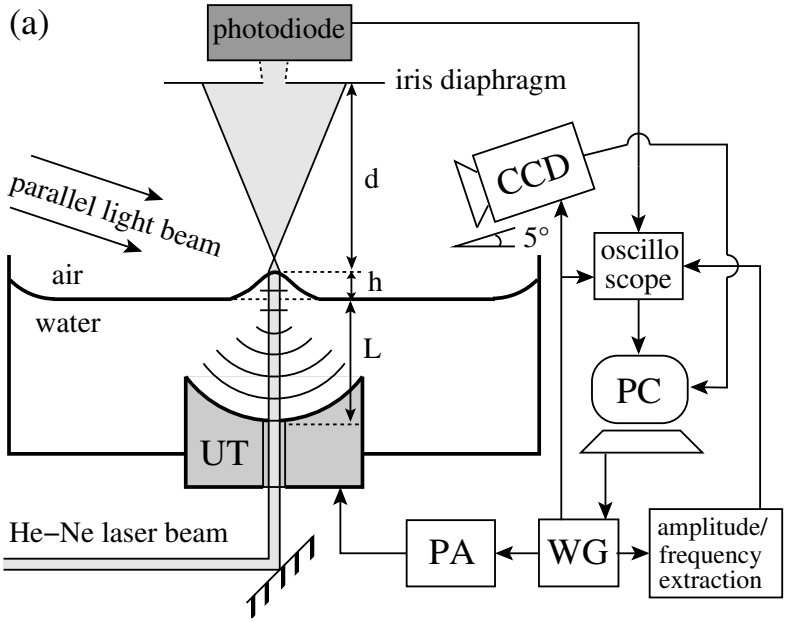

(b)
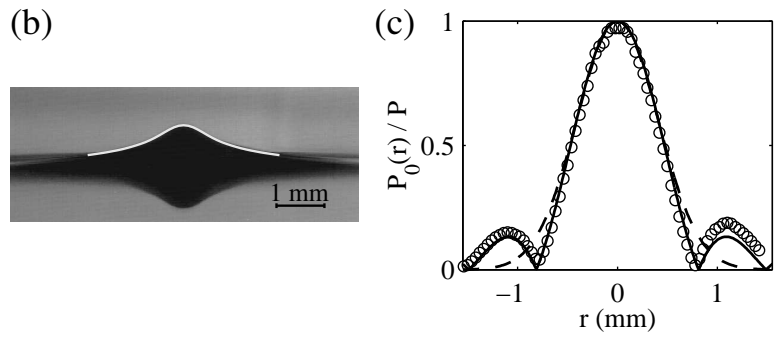

FIG. 1: (a) Experimental setup. (b) Picture of the steady free surface deformation (and of its reflection on the air-water interface) observed for a pressure amplitude $P=0.93 \mathrm{MPa}$ at focus and $f=2.46 \mathrm{MHz}$. Solid white line: predicted shape using Eq. (1). (c) Symbols: rescaled amplitude of the pressure field of the input beam measured in the focal plane. Solid line: prediction from Ref. [20]. Dashed line: Gaussian approximation.

the $i^{\text {th }}$ order Bessel function, $r$ the radius in cylindrical coordinates, and $\lambda=660 \mu \mathrm{m}$ is the acoustic wavelength at central frequency). Typical pressure amplitudes at focus are $P=0.04-1.4 \mathrm{MPa}$. The liquid free surface, located at focus $(L \simeq F)$, totally reflects the incident acoustic beam and is deformed by RP, which expresses as $\Pi(r)=\frac{2\left\langle p_{i}^{2}(r, t)\right\rangle_{t}}{\rho c^{2}}[21]$, where $p_{i}(r, t)=P_{i}(r) \cos (2 \pi f t)$ is the pressure field of the incident beam in the focal plane (i.e. of the input beam $p_{0}(r, t)$ combined with its multiple reflections incident on the surface), $\rho$ is the water density, and $c$ the sound speed in water. It is illuminated at grazing incidence by a parallel beam of white light, and the pictures of the surface deformation are captured at $50 \mathrm{fps}$ using a high-speed CCD camera triggered by the WG. For continuous and moderate acoustic intensities, the liquid surface deformations are stationary, bellshaped, and of diameter at half height comparable to the diameter at half maximum in energy of the acoustic beam, $650 \mu \mathrm{m}$, see Fig. $1 \mathrm{~b}$ and $\mathrm{c}$.

To evidence the acoustic bistability, we ramp $P^{2} \propto$ $\Pi(0)$ linearly up and down within $5 \mathrm{~s}$ and simultaneously measure the variations of the height $h$ of the surface deformation, defined in Fig. 1a, as a function of time. The

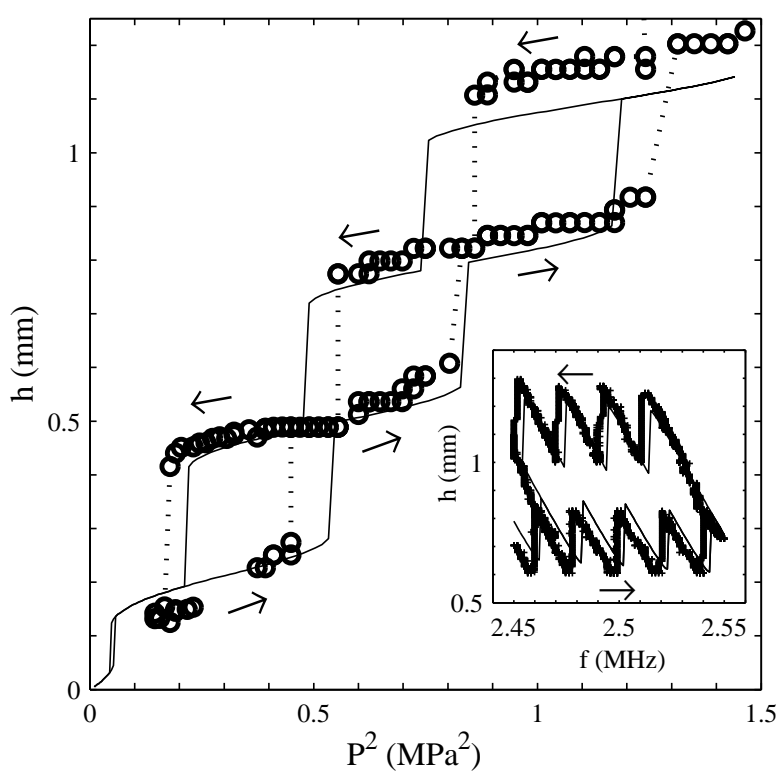

FIG. 2: Surface deformation height $h$ versus the square of the pressure amplitude $P$ at focus due to the input beam $(f=2.5 \mathrm{MHz})$. Symbols: experiment. Solid line: theoretical prediction using the model illustrated in Fig. 3. Inset: $h$ versus the acoustic frequency $f$ at $P=0.97 \mathrm{MPa}$. Symbols: experiment. Solid line: theoretical prediction.

variations of $h$ versus $P^{2}$ are plotted in Fig. 2. $h$ exhibits several hysteresis cycles. Above $h \simeq 1.4 \mathrm{~mm}$, irregular jetting and atomization-induced fog intermittently appear, a phenomenon usually called "acoustic fountain" [12].

To model the observed hysteresis, we consider only the coupling between the RP induced surface deformation and the condition for resonance of the compliant acoustic cavity. Since the measured characteristic timescale of relaxation of the surface deformation is a ten of milliseconds, the deformation evolves adiabatically during the $\mathrm{RP}$ ramps. Thus, its instantaneous axisymmetric shape $h(r)$ results from the balance between $\mathrm{RP}$ and the restoring effects of both gravity $g$ and surface tension $\sigma$, i.e. it verifies $\rho g h(r)-\sigma \kappa(r)=\Pi(r)$, where $\kappa(r)$ is the surface curvature. Assuming small deformations, i.e. $\left|h^{\prime}(r)\right| \ll 1$ and $h \ll z$, and taking $P_{i}(r)=P_{i} \exp \left(-r^{2} / \omega_{0}^{2}\right)$, where $\omega_{0}=0.86 \lambda$, for the pressure amplitude of the incident beam in the focal plane (see Fig. 1c), the surface shape reads [22]:

$$
h(r)=\frac{P_{i}^{2} \omega_{0}^{2}}{4 \rho c^{2}} \int_{0}^{\infty} J_{0}(k r) \frac{\exp \left(-\frac{k^{2} \omega_{0}^{2}}{8}\right)}{\rho g+\sigma k^{2}} k d k,
$$

and its height along the beam axis is :

$$
h=h(r=0)=\frac{P_{i}^{2} \omega_{0}^{2}}{8 \sigma \rho c^{2}} \exp \left(\frac{\rho g \omega_{0}^{2}}{8 \sigma}\right) E_{1}\left(\frac{\rho g \omega_{0}^{2}}{8 \sigma}\right),
$$

where $E_{1}$ is the 1-argument exponential function [22]. Fig. 1b illustrates the accuracy of this linear model. 


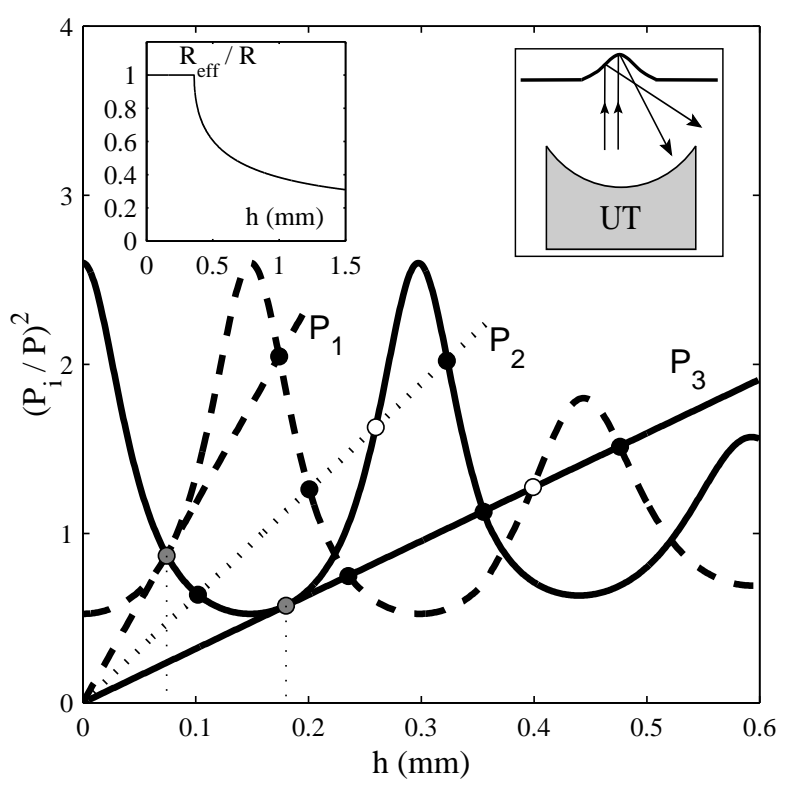

FIG. 3: Model for acoustic hysteresis. Curves: resonance curves of the cavity, represented by the square of the pressure amplitude $P_{i}$ at focus due to the incident beam rescaled by the pressure amplitude $P$ at focus due to the input beam versus the surface deformation height $h$ (Eq. (3)) for $2 L / \lambda$ half-integer, $f=2.4982 \mathrm{MHz}$ (dashed line), $2 L / \lambda$ integer, $f=2.5079 \mathrm{MHz}$ (solid line). Straight lines: linear relationship between $h$ and the radiation pressure (Eq. (2)) at $P_{1}=0.292 \mathrm{MPa}, P_{2}=0.400 \mathrm{MPa}, P_{3}=0.563 \mathrm{MPa}$. Black (white, gray) points correspond to stable (unstable, marginally stable) equilibrium of the free surface. Left inset: variation versus $h$ of the effective reflection coefficient of the UT $R_{\text {eff }}$ rescaled by $R$, see sketch and text. Right inset: sketch for the energy leak calculation.

On the other hand, we model the acoustic cavity bounded by the UT and the free liquid surface as a one dimensional Fabry-Pérot resonator. Considering that the incident acoustic energy is totally reflected by the flat part of the deformation close to the beam axis and that the reflected wave remains plane, acoustic resonance is expected to occur when $2(L+h)=n \lambda, n$ integer (see Fig. 1a). More precisely,

$$
P_{i}=\left|1-R R^{\prime} \exp \left(i \frac{4 \pi(L+h)}{\lambda}\right)\right|^{-1} P,
$$

where $R=-0.38 \pm 0.05, R^{\prime}=-1$ are the pressure reflection coefficients of the UT surface, of the air-water interface measured independently. However due to the curvature of the free surface, some acoustic energy leaks out of the cavity (see sketch in Fig. 3). This results in an effective reflection coefficient $R_{\text {eff }}$ estimated by determining numerically which vertically incident acoustic rays impinge the UT after reflection on a deformation of shape given by Eq. (1). The variation of $R_{\mathrm{eff}} / R$ versus $h$ is plotted in the inset of Fig. 3 .

Finally, as illustrated in Fig. 3, the deformation height can be graphically determined as the intersection of the reduced resonance curve $\left(P_{i} / P\right)^{2}(h)$ defined by Eq. (3) where $R$ is replaced by $R_{\text {eff }}(h)$, with the linear relationship $\left(P_{i} / P\right)^{2} \propto h / P^{2}$ deduced from Eq. (2). The resulting multistability leads to hysteresis as the slope of the straight line varies with $P$. The predicted hysteretic behavior of $h$ versus $P^{2}$, shown in Fig. 2, agrees quantitatively with the measurements. Note that, contrary to previous studies of bistability induced by optical forces $[2,3,5]$, the model involves no free parameter, since the acoustic path length of the undeformed resonator $2 L / \lambda$ is experimentally accessed through $\tau$, the round-trip time of flight of a wave train inside the cavity, measured independently: $2 L / \lambda=f \tau=128.60 \pm 0.08$ for the experiment reported in Fig. 2.

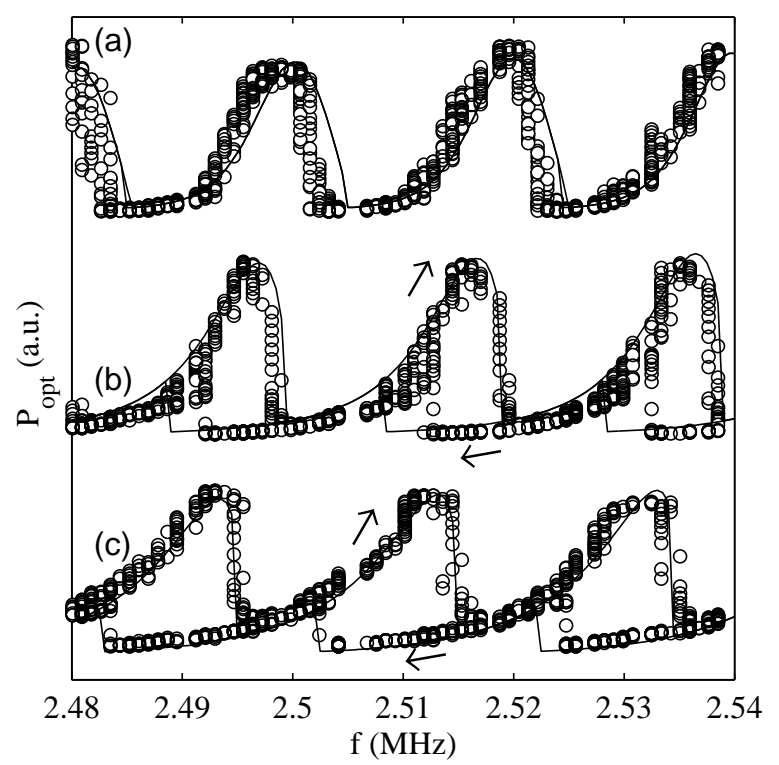

FIG. 4: Power $P_{\mathrm{opt}}$ of the laser beam refracted by the surface deformation versus acoustic frequency $f$. Symbols: experiment at (a) $P=0.19 \mathrm{MPa}$, (b) $P=0.36 \mathrm{MPa}$, (c) $P=0.48 \mathrm{MPa}$. Solid lines: theoretical prediction. Curves have been vertically shifted and rescaled for clarity.

Additionally, we investigated RP induced acoustic bistability by varying the frequency at fixed $P$, as is often done when bulk nonlinearities are studied [9]. We sweep the driving frequency $f$ linearly up and down within $5 \mathrm{~s}$ at constant voltage amplitude, and measure it using a digital counter (accuracy $1 \mathrm{kHz}$, sampling rate $50 \mathrm{~Hz}$ ). In this frequency ramp mode, the deformation height also exhibits hysteresis above a threshold in $P$ which depends on $2 L / \lambda$. More precisely, the above model predicts that the minimum height at which bistability occurs (marginal stability) varies from $74 \mu \mathrm{m}$ for $2 L / \lambda=n+1 / 2$ to $180 \mu \mathrm{m} \simeq \lambda / 4$ for $2 L / \lambda=n, n$ integer (gray points in Fig. 3). In order to detect such small deformations, we use the lens effect of the surface deformation on a laser beam, a technique recently developed for contactless surface tension measurements [23]. A Gaussian HeNe laser beam (beam waist $500 \mu \mathrm{m}$ ) passing vertically 
through the glass cylinder inserted in the UT hole is focused by the surface deformation and filtered through a diaphragm distant of $d$ from the surface, see Fig. 1a. Its power $P_{\mathrm{opt}}$ is measured using a linear photodetector. Assuming the liquid lens to be spherical, its focal length is $f_{\text {opt }}=\left(\kappa\left(n_{w}-1\right) / 2\right)^{-1}$ where $n_{w}$ is the water refractive index and $\kappa=\frac{1}{r} \frac{d}{d r}\left(r h^{\prime}\right)(0)$ is the linearized surface curvature on beam axis, of value $1.6 h / \omega_{0}^{2}$ deduced from Eq. (1). In the ray optics approximation, $P_{\text {opt }} \propto\left(d f_{\text {opt }}^{-1}-1\right)^{-2}$. As shown in Fig. 4, when $P$ is increased from small values, the resonance curves of the compliant cavity exhibit increasing asymmetry (curve a in Fig. 4), and ultimately show hysteresis (curve b). Using the refraction model presented above, our model of hysteresis can predict the variation of $P_{\text {opt }}$ versus $h$ in the frequency sweep mode. When the frequency is swept, the successive equilibrium surface heights can also be determined graphically in Fig. 3 by now mainly translating the cavity resonance curve (variable acoustic path length $2 L / \lambda$ ) while the straight line remains unchanged (fixed $P$ ). The measured and predicted values of $P_{\mathrm{opt}}$ were found to differ by a factor of typically 20, probably because (i) the laser beam slightly diverges, (ii) the beam intensity strongly differs from Gaussian after transmission through the roughly polished glass cylinder and (iii) the bell-shaped liquid lens induces spherical aberrations. Nevertheless, their dependence versus $f$ are qualitatively very similar, as shown in Fig. 4. Moreover, the predicted threshold in $P$ at which hysteresis occurs quantitatively agree with experiment. When $P$ is further increased, the hysteresis cycles broaden (curve c in Fig. 4). At large $P$, the height variations for increasing and decreasing frequency eventually split and give birth to a unique hysteresis cycle extending over the whole frequency span, as shown in the inset of Fig. 2, where $h$ was measured through image analysis. This behavior is also shown to be quantitatively well described by our model.

To conclude, the multiple-order hysteresis of a compliant acoustic cavity formed between a spherical UT and a liquid free surface located at its focus was observed for the first time and was accurately described using a one dimensional model of Fabry-Pérot resonator assuming acoustic RP to be the only coupling between the cavity and the acoustic field. Unlike former investigations of optical RP, the variation of the cavity length, on which bistability relies, is directly evidenced. This study demonstrates that, at least in stationary and one dimensional situations, the coupling between a wave and a resonator through the RP can be described in a universal manner for electromagnetic and acoustic waves. Finally, we note that electromagnetic RP was recently shown to trigger a parametric oscillatory instability $[5,6,24]$ that may limit the miniaturization of optical resonators as well as the performances of GWD [25]. Investigating this instability in acoustics would extend to dynamical behaviors our study on the universality of the effects of RP.

The authors thank A. Nicolas and O. Misère for preliminary experiments, and J. Plantard and S. Cassagnere for technical assistance. This work was supported by Centre National de la Recherche Scientifique, Université Bordeaux 1, and Conseil Régional d'Aquitaine under contract $\mathrm{n}^{\circ} 2005-1101010 \mathrm{~A}$.
[1] H. M. Gibbs, Optical bistability: controlling light with light, Academic, 1985.

[2] A. Dorsel, J. D. Mc Cullen, P. Meystre, E. Vignes, and H. Walter, Phys. Rev. Lett. 51, 1550 (1983).

[3] A. Gozzini, F. Maccarone, F. Mango, I. Longo, ans S. Barbarino, J. Opt. Soc. Am. B 2, 1841 (1985).

[4] R. S. Tucker, D. M. Baney, W. V. Sorin, C. A. Flory, IEEE J. Sel. Top. Quantum Electron. 8, 88 (2002).

[5] C. Höhberger Metzger and K. Karrai, Nature 432, 1002 (2004).

[6] T. J. Kippenberg, H. Rokhsari, T. Carmon, A. Scherer, and K. J. Vahala, Phys. Rev. Lett. 95, 033901 (2005).

[7] B. S. Sheard, M. B. Gray, C. M. Mow-Lowry, D. E. McClelland, and S. E. Whitcomb, Phys. Rev. A 68, 051801(R) (2004).

[8] A. Pai, S. V. Dhurandhar, P. Hello, and J. L. Vinet, Eur. Phys. J. D 8, 333 (2000).

[9] C. C. Lawrenson, B. Lipkens, T. S. Lucas, D. K. Perkins, and T. W. Van Doren, J. Acoust. Soc. Am. 104, 623 (1998).

[10] A. Boudaoud, Y. Couder, and M. Ben Amar, Eur. Phys. J. B 9, 159 (1999).

[11] Y. A. Ilinskii, B. Lipkens, and E. A. Zabolotskaya, J. Acoust. Soc. Am. 109, 1859 (2001).

[12] R. W. Wood and A. L. Loomis, Phil. Mag. S. 7 4, 417 (1927).
[13] G. Hertz and H. Mende, Z. Physik 114, 354 (1939).

[14] E. H. Brandt, Nature 413, 474 (2001).

[15] M. J. Marr-Lyon, D. B. Thiessen, and P. L. Marston, Phys. Rev. Lett. 86, 2293 (2001).

[16] C. Cinbis and B. T. Khuri-Yakub, Rev. Sci. Instrum. 63, 204 (1992).

[17] M. Fatemi and J. F. Greenleaf, Science 280, 82 (1998).

[18] M. Mansuripur, Opt. express 12, 5375 (2004).

[19] G.T. Silva, S. Chen, and L. P. Viana, ArXiv:physics 0601016 (2006).

[20] G. S. Kino, Acoustic waves, devices, imaging and analog signal processing, Prentice-Hall, 1987.

[21] B. Chu and R. E. Apfel, J. Acoust. Soc. Am. 72, 1673 (1982).

[22] A. Casner and J.P. Delville, Phys. Rev. Lett. 87, 054503 (2001).

[23] K. Sakai, D. Mizuno, and K. Takagi, Phys. Rev. E 63, 046302 (2001).

[24] T. Carmon, H. Rokhsari, T. J. Kippenberg, and K. J. Vahala, Phys. Rev. Lett. 94, 223902 (2005). H. Rokhsari, T. K. Kippenberg, T. Carmon, and K. J. Vahala, Optics Express 13, 5293 (2005).

[25] V. B. Braginsky, S. E. Strigin, S. P. Vyatchanin, Phys. Lett. A 305, 111 (2002). 